\title{
Microscopic Dynamics in a Strongly Interacting Bose-Einstein Condensate
}

\author{
N. R. Claussen, E. A. Donley, S. T. Thompson, and C. E. Wieman \\ JILA, National Institute of Standards and Technology and the University of Colorado, \\ and the Department of Physics, University of Colorado, Boulder, Colorado 80309-0440
}

(Dated: October 31, 2018)

\begin{abstract}
An initially stable ${ }^{85} \mathrm{Rb}$ Bose-Einstein condensate (BEC) was subjected to a carefully controlled magnetic field pulse in the vicinity of a Feshbach resonance. This pulse probed the strongly interacting regime for the condensate, with calculated values for the diluteness parameter $\left(n a^{3}\right)$ ranging from 0.01 to 0.5 . The field pulse was observed to cause loss of atoms from the condensate on remarkably short time scales $(\geq 10 \mu \mathrm{s})$. The dependence of this loss on magnetic field pulse shape and amplitude was measured. For triangular pulses shorter than $1 \mathrm{~ms}$, decreasing the pulse length actually increased the loss, until extremely short time scales (a few tens of microseconds) were reached. Such time scales and dependencies are very different from those expected in traditional condensate inelastic loss processes, suggesting the presence of new microscopic BEC physics.
\end{abstract}

PACS numbers: 03.75.Fi, 05.30.Jp, 32.80.Pj, 34.50.-s

The self-interaction strength that determines most of the properties of a Bose-Einstein condensate (BEC) is characterized simply by the s-wave scattering length, $a$ [1]. Near a Feshbach resonance [2], the scattering length depends strongly on the magnetic field. This property has been used to vary the self-interaction energy in BEC 3, 因, 5, 6, 7. In particular, we have used the Feshbach resonance to create stable ${ }^{85} \mathrm{Rb}$ condensates 5 . We also varied the scattering length and studied the effect on the condensate - most notably its collapse when the scattering length was made negative [6, 7].

Here we discuss BEC behavior in the positive $a$ region near the ${ }^{85} \mathrm{Rb}$ Feshbach resonance, where the selfinteraction is large and repulsive. We used rapid magnetic field variations to probe the strongly interacting regime in the condensate and to investigate the possibility of collisional coupling between pairs of free atoms and bound molecules [8, 9, 10, 11, 12, 13. . We changed the magnetic field to approach the Feshbach resonance from above, which causes the positive scattering length to increase. As $n a^{3}$ becomes comparable to or larger than one, the customary mean-field description of the dilute gas BEC, which assumes no correlations between the atoms, breaks down. Correlations between the atoms become increasingly important and a BEC with sufficiently large $n a^{3}$ will eventually reach a highly correlated state such as that found in a liquid. We are interested in the nature of this transition to a highly correlated state and the time scale for the formation of the condensate correlations. In addition to such interesting physics, our time dependent experiments also allow us to probe the coupling between atomic and molecular BEC states that are degenerate at the Feshbach resonance. Several authors have proposed mechanisms for transferring atom pairs into molecules using time-dependent magnetic fields near resonance [8, 9, 10, 11, 12, 13]. To investigate such physics we examined the response of the condensate when we briefly approached the resonance by applying a short magnetic field pulse (much shorter than the period for collective excitations). The most obvious feature of the $\mathrm{BEC}$ response was a loss of atoms that increased when we made our pulses shorter, until the loss became very large for very short pulses.

Bose-Einstein condensates always disappear if one waits long enough. The dominant loss process was found to be three-body recombination into molecules [14, 15, with a time dependence characterized by a simple rate equation and rate constant. All previous observations of condensates are consistent with a mean-field description that includes such a density-dependent loss process. In the vicinity of a Feshbach resonance the decay rates have been seen to increase dramatically 4,0 , 16], becoming so large that various novel coherent conversion processes have been put forth to explain their magnitude [10, 11, 12. However, the observations in Refs. [4, 目, 16] always revealed that the more time spent near the Feshbach resonance, the greater the loss. This is still consistent with the basic picture of a mean-field with some inelastic loss term, albeit a very large one. Here we report losses occurring on very fast time scales even when we remain some distance from the resonance, and we see that shorter and more rapid pulses lead to a greater loss than longer, slower pulses. This behavior contrasts dramatically with the conventional picture presented above, and indicates the presence of novel BEC physics.

To study BEC dynamics we first formed $\mathrm{a}^{85} \mathrm{Rb}$ condensate, following the procedure given in Ref. [5]. A sample of ${ }^{85} \mathrm{Rb}$ atoms in the $\mathrm{F}=2 \mathrm{~m}_{F}=-2$ state was evaporatively cooled in a cylindrically symmetric magnetic trap $\left(\nu_{\text {radial }}=17.5 \mathrm{~Hz}, \nu_{\text {axial }}=6.8 \mathrm{~Hz}\right)$. The magnetic field at completion of evaporation was $162.3 \mathrm{G}$, corresponding to a scattering length of $200 a_{0}$. Typically, the cooled sample had $N_{0}=16,500$ BEC atoms and fewer than 1000 noncondensed atoms. The magnetic field was then ramped adiabatically (in $800 \mathrm{~ms}$ ) to $\sim 166 \mathrm{G}$ where the scattering length was positive but nearly zero 17, and 
the BEC assumed the shape of the harmonic trap ground state.

We next applied a short magnetic field pulse (duration $<1 \mathrm{~ms}$ ) so that the field briefly approached a value moderately close to the Feshbach resonance at $\sim 155 \mathrm{G}$ (see Fig. 1). We used destructive absorption imaging to look at the number of atoms remaining in the condensate. This experiment was repeated with a variety of differently shaped magnetic field pulses.

We found that the magnetic trap must be turned off and the condensate spatial size must be significantly larger than our resolution limit to obtain the most sensitive and accurate measurements of number [7. To expand the BEC after the short pulse, we ramped in $5 \mathrm{~ms}$ to $\sim 157 \mathrm{G}\left(a=1900 a_{0}\right)$, and then held at that field for $7 \mathrm{~ms}$. The mean-field repulsion during the ramp and hold times decreased the density by about a factor of 30, then we rapidly turned off the trap and imaged the cloud. The density decrease avoided density-dependent loss that we have observed during the trap turn-off [ 5,16 .

Significant number loss from the BEC was observed for pulses lasting only a few tens of microseconds. The loss was accompanied by the generation of a "burst" of relatively hot $(\sim 150 \mathrm{nK})$ atoms that remained in the trap, as in Ref. [7]. The few thousand burst atoms represented a fairly small fraction of the total number lost from the BEC and so in this Letter we have focused only on the number in the condensate remnant. The burst will be the subject of future work.

To study the remarkably short time scales for the loss, we designed a low-inductance, high current auxiliary electromagnetic coil. The coil current was supplied by a capacitor bank that was charged to $580 \mathrm{~V}$, then discharged through the coil at a rate controlled by a transistor. Our goal was to create a perfect trapezoidal magnetic field pulse with adjustable but identical rise and fall times and a hold time during which the field was constant. To compensate for observed mutual inductance effects with other coils in the apparatus, we empirically determined the auxiliary current pulse shape needed to produce a total magnetic field pulse that closely approximated our ideal, as shown in Fig. 1. The presence of induced currents limited the maximum ramp speed to $d B / d t=1 \mathrm{G} / \mu \mathrm{s}$, and the magnetic field uncertainty was 1 part in $10^{3}(0.16 \mathrm{G})$.

We first examined the BEC loss for trapezoidal field pulses as a function of the hold time (see Fig. 2). Using a linear rise and fall time of $12.5 \mu \mathrm{s}$, we observed BEC number loss of $10-20 \%$ when the hold time was set to zero (triangular pulse). The number of BEC atoms then showed a smooth exponential decrease as hold time was increased. Surprisingly, when we reduced the initial BEC density by more than a factor of 2 , the time constant was nearly unchanged. For the low[high] density data, the value of the time constant was over 2[1] orders of magnitude shorter than predicted by our previous inelastic

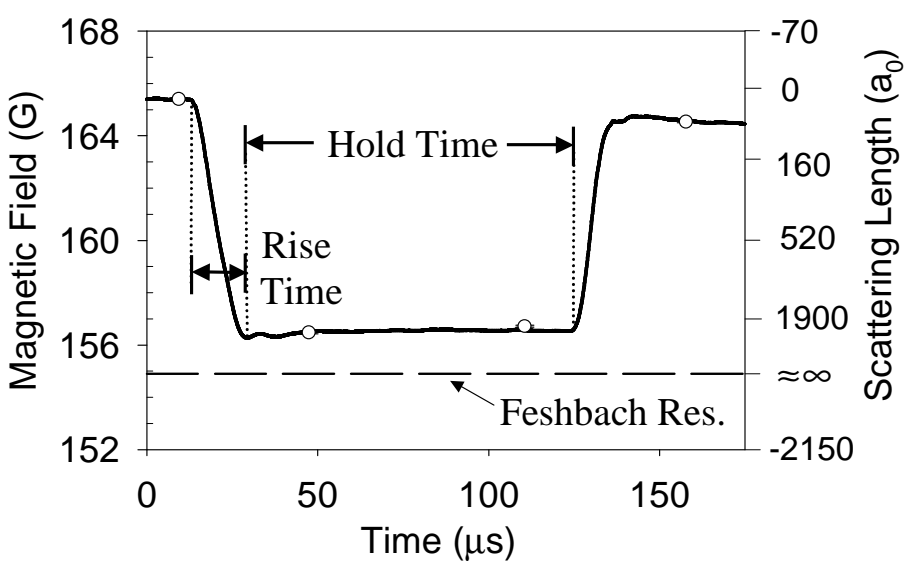

FIG. 1: The magnetic field vs. time for a typical auxiliary coil pulse. The solid line is the field calculated from measurements with calibrated Hall-effect current sensors on each electromagnet coil. On the right vertical axis, the corresponding variation in scattering length is shown. The open circles are independent checks of the magnetic field obtained from the resonant frequency for a $10 \mu \mathrm{s} R$ radiation pulse that drives atoms to the $\mathrm{m}_{F}=-1$ spin state (error bars are smaller than the points). The dashed line shows the position of the Feshbach resonance, where the scattering length becomes infinite. The field variation on the peak of the pulse was typically $\Delta \mathrm{B} \lesssim 0.1 \mathrm{G}$.

decay measurements with cold thermal clouds 18 .

We next measured how the loss depended on the rise/fall time of the pulse for a variety of pulse amplitudes and hold times (Figs. 3 and 4). We varied the rise time from $12.5 \mu \mathrm{s}$ to $250 \mu \mathrm{s}$ and changed the hold time at the pulse peak from $1 \mu$ s to $100 \mu \mathrm{s}$. In addition, the amplitude was varied to examine fields from $158.0 \mathrm{G}$ $\left(a=1100 a_{0}\right)$ to $156.0 \mathrm{G}\left(a=4000 a_{0}\right)$. Here we list the corresponding scattering lengths that we have observed by slowly adjusting the magnetic field, as in Ref. [0. The scattering length was calculated from the equation: $a(B)=a_{b g}\left(1-\Delta /\left(B-B_{0}\right)\right)$, where $\Delta=11.0(4) \mathrm{G}$ is the width of the resonance, $B_{0}=154.9(4) \mathrm{G}$ is the resonant magnetic field, and $a_{b q}=-450(3) a_{0}$ is the background scattering length 19. For the range of magnetic fields examined here, the initial value of the diluteness parameter varied from $n a^{3}=0.01$ for $a=1100 a_{0}$ to $n a^{3}$ $=0.5$ for $a=4000 a_{0}$.

The number remaining in the BEC after the pulse vs. pulse rise time for a variety of different hold times is shown in Fig. 3. For hold times $\mathrm{t}_{\text {hold }} \leq 15 \mu \mathrm{s}$, there is an initial decrease in $\mathrm{N}_{r e m}$ as the rise time increases. Then the slope changes and fewer atoms are lost for longer rise times. All of the hold time data display this upward slope over some range, but the range is largest for the $100 \mu$ s hold time. The increase in remnant number for longer rise time provides clear evidence that the loss is not conventional inelastic decay that is characterized by a rate constant. It is interesting that the short hold 


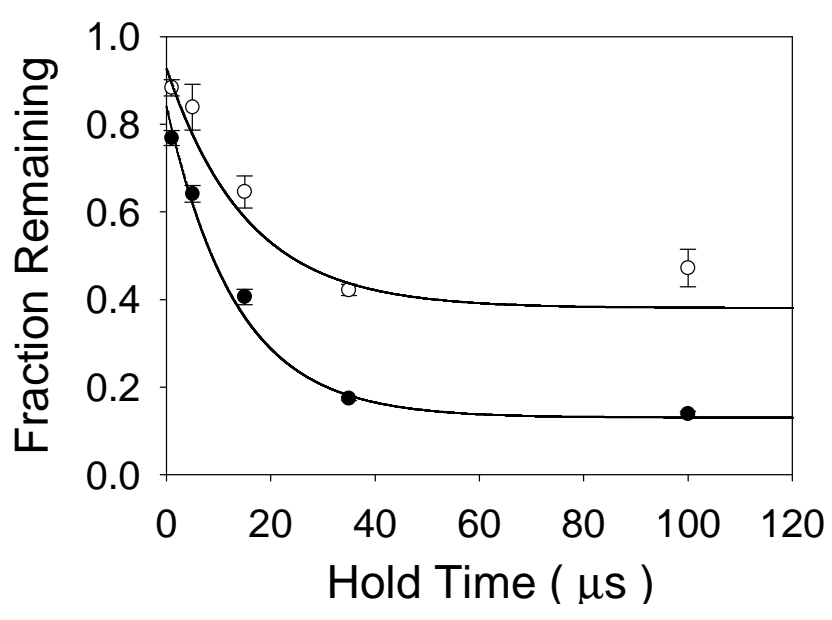

FIG. 2: Fraction of BEC remaining vs. pulse hold time. The pulse rise/fall time was $12.5 \mu \mathrm{s}$ and the magnetic field during the hold was $156.7 \mathrm{G}\left(2300 a_{0}\right)$. Number decay was measured for two different initial densities: $\langle n\rangle=1.9 \times 10^{13} \mathrm{~cm}^{-3}$ $(\bullet)$ and $\langle n\rangle=0.7 \times 10^{13} \mathrm{~cm}^{-3}(\circ)$. Fitting the data to exponential functions (solid lines) gave time constants of 13.2(4) $\mu \mathrm{s}$ and 15.4(14) $\mu \mathrm{s}$. Thus, reducing the density by a factor of 2.6 caused an increase of only $17(11) \%$ in the time constant.

time data show a distinct minimum in $\mathrm{N}_{r e m}$ vs. rise time, which shifts toward shorter rise times as the hold time is increased.

In Fig. 4 we display the remnant number vs. ramp time for triangular magnetic field pulses ( $1 \mu$ s hold $)$. The remnant number is shown for various pulse amplitudes. For all cases, $\mathrm{N}_{r e m}$ is large at the shortest rise time and then decreases with rise time until it reaches a minimum. Then longer rise times cause $\mathrm{N}_{r e m}$ to increase over a time scale of tens of microseconds. The rise time that induces maximal loss becomes longer as the pulses come closer to resonance.

Conventional condensate loss is characterized by a rate constant for a density-dependent decay process, and thus the loss increases monotonically with time. Near a Feshbach resonance the rate constants have been observed to increase enormously [4, 目, 16], but nevertheless, a longer time spent near the peak, or equivalently a slower ramp getting over it, resulted in greater loss. In contrast, we have measured an increase in the loss when the ramp time is decreased, which reveals the existence of previously unexplored BEC physics. Of course, the above interpretation of our data would be modified if the density of the sample was changing due to the rapid increase in the mean-field interaction, but the characteristic time for such readjustments in cloud shape is far longer (of order $\left.1 /\left(2 \nu_{\text {radial }}\right)=29 \mathrm{~ms}\right)$ than the time scales we considered here. For example, using a simple analytic model based on the Gross-Pitaevskii equation [22], we calculate that for a $250 \mu \mathrm{s}$ ramp to $\mathrm{B}_{\text {final }}=156.0 \mathrm{G}\left(4000 a_{0}\right)$, the change in mean-field energy causes the density to

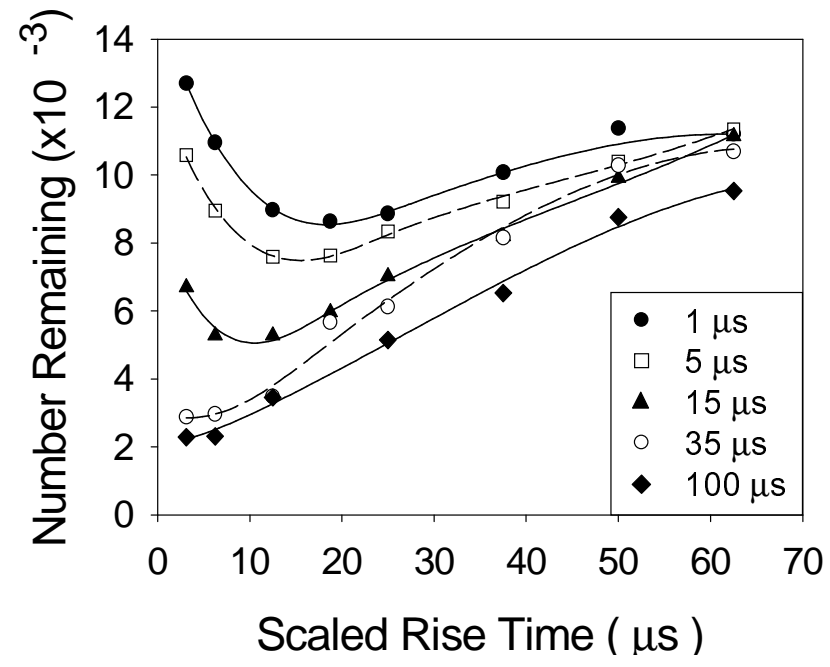

FIG. 3: Dependence of remnant BEC number on pulse rise/fall time for various hold times (see legend) with $N_{0}=16,500\left(\langle n\rangle_{0}=1.9 \times 10^{13} \mathrm{~cm}^{-3}\right)$. For the majority of the data points, the symbol is larger than the statistical error bar (not shown). The lines are spline fits to guide the eye. The abscissa was multiplied by a factor of $1 / 4$ to show the time required to ramp from $75 \%$ to $100 \%$ of the pulse amplitude. This scaling reflects the observed fact that most of the loss occurred at fields closest to the Feshbach resonance. The magnetic field during the hold time was $156.7 \mathrm{G}\left(2300 a_{0}\right)$.

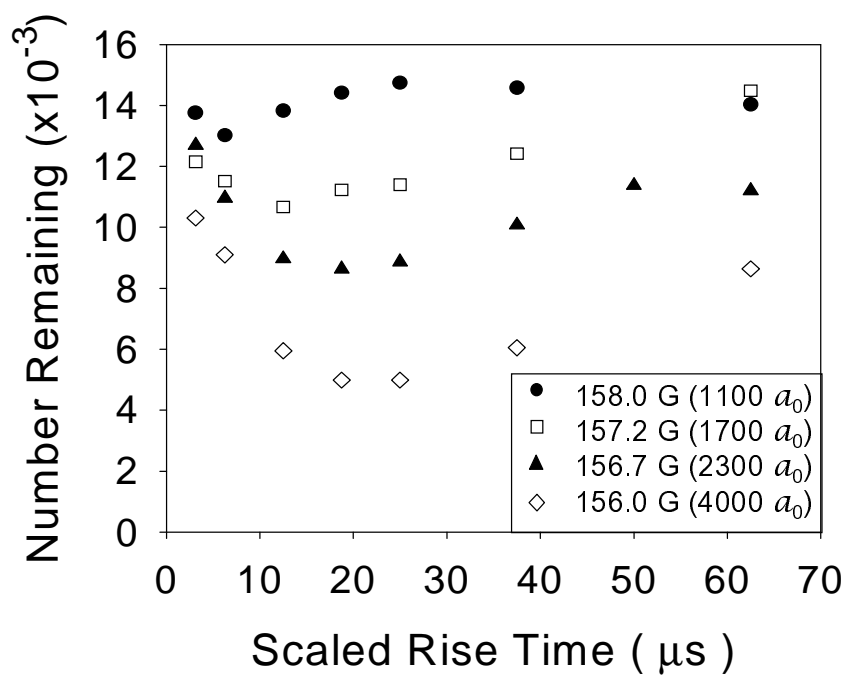

FIG. 4: Rise time dependence of BEC number for different triangular pulse amplitudes. The pulse hold time was fixed at $1 \mu$ s for these data and $\mathrm{N}_{0}=16,600$. Data points are larger than the error bars.

decrease by only $1 \%$ from its initial value. Thus, the observed dependencies on rise time must reflect microscopic physics in the BEC and not any macroscopic changes in the shape of the condensate.

The response of the BEC remnant to magnetic field ramps, observed in Fig. 4, is qualitatively similar to what 
one would expect in a Landau-Zener (L-Z) model 23, 24, of an avoided crossing of two states with linear Zeeman shifts, if the second state were undetectable. In our case, the obvious candidate for this second state is the Feshbach resonance bound state corresponding to a diatomic molecule. With a L-Z avoided crossing, the behavior when the field approaches and then backs away from the crossing point with a triangular pulse is qualitatively similar to the more familiar and analytically soluble case 25] of a linear field ramp that goes from far above the crossing to far below and then back again. In both cases, and as seen in Fig. 4, the L-Z model predicts a steep rise from zero in the transition probability as the length of the ramp increases from zero (diabatic limit). As the ramp time increases further, the transition probability reaches a maximum - where the time derivative of the relative energy matches the square of the coupling strength and then slowly decreases to zero as the ramp approaches the adiabatic limit. We found that the L-Z model does a rather bad job at reproducing any more quantitative features of the data however, even when we allowed the coupling strength and relative magnetic moment to be arbitrary parameters in our numerical simulations [26].

Nevertheless, it seems likely that some atoms are being converted to another state (possibly molecular) by nonadiabatic mixing, although the process is more complicated than a simple Landau-Zener avoided crossing picture. In future experiments, we plan to further investigate the time response of the BEC loss using asymmetric pulses and double pulses with variable spacing. We will explore the burst production process and attempt to determine the fate of the lost atoms.

We would like to acknowledge contributions from the entire JILA BEC/degenerate Fermi gas collaboration and helpful discussions with L. Pitaevskii and E. Cornell. S. Thompson acknowledges the support of the AROMURI Fellowship Program. This work was also supported by ONR and NSF.

[1] F. Dalfovo et al., Rev. Mod. Phys. 71, 463 (1999).
[2] W. C. Stwalley, Phys. Rev. Lett. 37, 1628 (1976); E. Tiesinga et al., Phys. Rev. A 46, R1167 (1992).

[3] S. Inouye et al., Nature, 392, 151 (1998).

[4] J. Stenger et al., Phys. Rev. Lett. 82, 2422 (1999).

[5] S. L. Cornish et al., Phys. Rev. Lett. 85, 1795 (2000).

[6] J. L. Roberts et al., Phys. Rev. Lett. 86, 4211 (2001).

[7] E. A. Donley et al., Nature 412, 295 (2001).

[8] E. Timmermans et al., Phys. Rep. 315, 199 (1999); E. Timmermans et al., Phys. Rev. Lett. 83, 2691 (1999).

[9] P. D. Drummond, K. V. Kheruntsyan, and H. He, Phys. Rev. Lett., 81, 3055 (1998).

[10] F. A. van Abeelen and B. J. Verhaar, Phys. Rev. Lett. 83, $1550(1999)$.

[11] V. A. Yurovsky et al., Phys. Rev. A, 60, R765 (1999).

[12] F. H. Mies, E. Tiesinga, and P. S. Julienne, Phys. Rev. A, 61, 022721-1 (2000).

[13] M. Holland, J. Park, and R. Walser, Phys. Rev. Lett., 86, 1915 (2001).

[14] E. A. Burt et al., Phys. Rev. Lett. 79, 337 (1997).

[15] C. J. Myatt, PhD Thesis, University of Colorado (1997).

[16] J. L. Roberts, PhD Thesis, University of Colorado (2001), available at http://jilawww.colorado.edu/www/sro/thesis/.

[17] J. L. Roberts et al., Phys. Rev. A, 64, 024702-1 (2001).

[18] J. L. Roberts et al., Phys. Rev. Lett. 85, 728 (2000).

[19] The values for $\Delta$ and $B_{0}$ were taken from our previous work [17, while $a_{b g}$ came from Ref. 20]. Although this value for $a_{b g}$ disagrees somewhat with our measurements in Ref. [17], we use it because of the substantially smaller uncertainty stated in Ref. [21].

[20] S. J. J. M. F. Kokkelmans, private communication.

[21] E. G. M. van Kempen et al., Phys. Rev. Lett. 88, 093201/1-4 (2002).

[22] V. M. Pérez-García et al., Phys. Rev. A 56, 1424 (1997).

[23] L. D. Landau, Phys. Z. Sowjetunion, 2, 46, (1932).

[24] C. Zener, Proc. R. Soc. London Ser. A137, 696 (1932).

[25] L. D. Landau and E. M. Lifshitz, Quantum Mechanics: Non-relativistic Theory, 2nd ed. (Pergamon, New York, 1965), pp.322-330.

[26] See, for instance, J. R. Rubbmark et al., Phys. Rev. A 23, 3107 (1981). 